\title{
Differential transcriptional response to nonassociative and associative components of classical fear conditioning in the amygdala and hippocampus
}

\author{
Michael B. Keeley, ${ }^{1}$ Marcelo A. Wood, ${ }^{1,4}$ Carolina Isiegas, ${ }^{1}$ Joel Stein, ${ }^{1}$ Kevin Hellman, ${ }^{3}$ \\ Sridhar Hannenhalli, ${ }^{2}$ and Ted Abel $^{1,5}$ \\ ${ }^{1}$ Department of Biology and ${ }^{2}$ Department of Genetics, University of Pennsylvania, Philadelphia, Pennsylvania 19104, USA; \\ ${ }^{3}$ Committee on Neurobiology in the Department of Neurobiology, Pharmacology and Physiology, University of Chicago, \\ Chicago, Illinois 60637, USA
}

\begin{abstract}
Classical fear conditioning requires the recognition of conditioned stimuli (CS) and the association of the CS with an aversive stimulus. We used Affymetrix oligonucleotide microarrays to characterize changes in gene expression compared to naive mice in both the amygdala and the hippocampus $30 \mathrm{~min}$ after classical fear conditioning and 30 min after exposure to the CS in the absence of an aversive stimulus. We found that in the hippocampus, levels of gene regulation induced by classical fear conditioning were not significantly greater than those induced by CS alone, whereas in the amygdala, classical fear conditioning did induce significantly greater levels of gene regulation compared to the CS. Computational studies suggest that transcriptional changes in the hippocampus and amygdala are mediated by large and overlapping but distinct combinations of molecular events. Our results demonstrate that an increase in gene regulation in the amygdala was partially correlated to associative learning and partially correlated to nonassociative components of the task, while gene regulation in the hippocampus was correlated to nonassociative components of classical fear conditioning, including configural learning.
\end{abstract}

Although post-translational modification of existing molecules may be sufficient for the storage of short-term memory for conditioned fear, long-term memory is mediated by changes in gene expression induced by the activation of intracellular signaling pathways (Abel and Lattal 2001), and these occur at precise times after training (Bernabeu et al. 1997; Bourtchouladze et al. 1998). Characterizing transcriptional regulation during memory formation is therefore a key challenge for understanding the mechanism supporting long-term memory storage. Recent studies have revealed that learning induces a complex reprogramming of gene expression involving the regulation of many genes. For example, hippocampal gene expression has been examined following training for eye-blink conditioning (Cavallaro et al. 2001; Donahue et al. 2002), spatial navigation (Cavallaro et al. 2002; Leil et al. 2002, 2003), swim-escape (Irwin 2001), spatial discrimination (Robles et al. 2003), passive avoidance (Robles et al. 2003), and classical fear conditioning (Levenson et al. 2004). There was little agreement in the lists of genes identified after these various forms of training, possibly because of the variation in the type of training that occurred and the range of time points examined.

Although microarrays have not previously been used to examine gene expression in the amygdala following behavioral manipulation, several genes are known to be differentially regulated in the amygdala during memory storage. For example, Fos is induced in the rat amygdala following both conditioned and unconditioned fear (Campeau et al. 1991), and Fos and Egr-1 are up-regulated following contextual fear conditioning (Campeau et al. 1991; Rosen et al. 1998). When genes known to be up-

\footnotetext{
${ }^{4}$ Present address: Department of Neurobiology and Behavior, University of California, Irvine, CA.

${ }^{5}$ Corresponding author.

E-mail abele@sas.upenn.edu; fax (215) 898-8780.

Article published online ahead of print. Article and publication date are at http://www.learnmem.org/cgi/doi/10.1101//m.86906.
}

regulated by seizure were examined in the amygdala following cued fear conditioning, Fos, EGR-1, Jun, NF1, Gphn, Nrgn, Nr4a1, Actn1, $16 c 8$, and $C d h 2$ were all found to be up-regulated (Ressler et al. 2002). In another study, subtractive hybridization was used to identify 12 genes regulated in the amygdala by classical fear conditioning (Stork et al. 2001). Thus, the amygdala has been demonstrated to undergo complex changes in gene expression following behavior that requires further characterization.

The present study focuses on gene regulation in both the amygdala and hippocampus following classical fear conditioning. Classical fear conditioning provides a means to correlate the degree of gene regulation in a particular brain region with the proposed role of that brain region in learning and memory. The amygdala has been proposed to function in the associative component of classical fear conditioning, while the hippocampus has been proposed to support the configural component of the task (Maren 2001). Circuitry in the amygdala may be modified during classical fear conditioning such that a subsequent appropriate sensory input would cause activity patterns in amygdala via the thalamus that activate areas of the brainstem responsible for innate fear responses (Pare et al. 2004). In the hippocampus, modification of the synaptic circuitry has been shown to play a role in behavior, such as the modification of place cell fields (Sharp et al. 1985; Jeffery and Hayman 2004). In the processing of contextual fear conditioning, the hippocampus is likely to store a conjunctive representation of the context, as demonstrated by experiments combining pre-exposure to the training context and protein synthesis inhibitors (Barrientos et al. 2002).

Like neurons that act in networks to relay information across brain regions, genes are likely to act coordinately in functional networks to support cellular processes underlying longterm memory storage. The goal of this experiment was therefore to examine these two brain regions and generate hypotheses regarding the similarities and differences in gene regulation re- 
sponsible for these similarities and differences in the regional response to behavioral manipulation. To accomplish this, we used Affymetrix mouse genome U74v2A microarrays to examine gene expression patterns, comparing mice that were naive, exposed to conditioned stimuli (CS), or conditioned to fear those CS. We used statistical methods to identify regulated genes and computational methods to predict underlying regulatory mechanisms.

\section{Results}

We examined the transcriptional changes in the amygdala and the hippocampus $30 \mathrm{~min}$ after classical fear conditioning. Fearconditioned (FC) mice were conditioned to fear a novel context plus tone by a single presentation of these CS paired with a mild footshock. CS-exposed (CS) mice received exposure to the CS without shock. Naive $(\mathrm{N})$ mice received no behavioral manipulation on the day of training (Fig. 1A). Training consisted of a 3-min exposure to the conditioning chamber, a 30-sec exposure to tone, and (for FC training only) a 2-sec, $1.5-\mathrm{mA}$ footshock. Classical conditioning produced robust fear upon exposure to the CS $24 \mathrm{~h}$ after training. FC-trained mice demonstrated robust freezing behavior $(58.4 \% \pm 3.4 \%)$ compared to CS mice $(4.5 \% \pm 1.2 \%)$ in response to the CS presented $24 \mathrm{~h}$ later (Fig. 1B). Thus classical fear conditioning produced evidence of robust learning.

\section{Microarray analysis reveals different expression patterns in amygdala and hippocampus}

The groups of mice used for microarray analysis were trained in parallel with those tested behaviorally. The expression profiles in the amygdala and hippocampus were examined $30 \mathrm{~min}$ after training, corresponding to the timing of immediate-early gene induction. Samples produced from either four amygdalae from two mice or two hippocampi from one mouse were measured on individual Affymetrix MGU74v2A microarrays (amygdala, $\mathrm{N}$ : $n=10$ arrays, 20 mice; FC: $n=6$ arrays, 12 mice; CS: $n=6$ arrays, 12 mice; hippocampus, N: $n=10$ arrays, 10 mice; FC: $n=8$ arrays, 8 mice; CS: $n=8$ arrays, 8 mice). Intensity scores were determined by Robust Multiarray Average, and MvsA plots were examined to ensure there was no bias due to signal intensity (data not shown).

Volcano plots were produced to gain perspective on global trends in our data (Fig. 2). In these pairwise group comparisons,
A

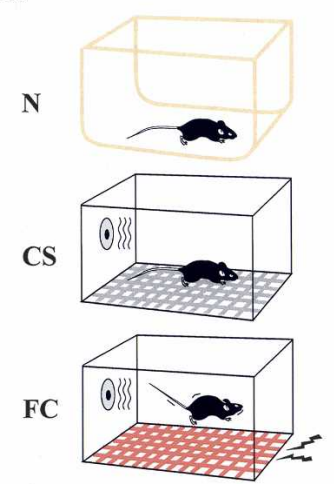

B

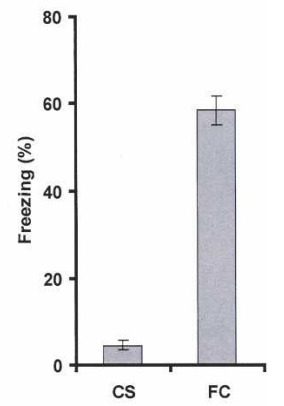

Figure 1. Classical fear conditioning. (A) Mice were left in their home cage $(N)$, exposed to the conditioned stimulus (CS), or conditioned to fear the CS by coadministration of footshock (FC) and were then dissected 30 min later or tested for conditioned fear at $24 \mathrm{~h}$. (B) Mice that were FC-trained demonstrated robust associative learning by exhibiting freezing behavior during $\sim 60 \%$ of the re-exposure to the training environment at $24 \mathrm{~h}$. CS-trained mice demonstrated only baseline levels of freezing behavior. contrast $P$-values from mixed model ANOVA are plotted against the fold change, expressed as the base two log. The volcano plots demonstrate that the most significantly up-regulated genes were more statistically significant compared to down-regulated genes because each scatterplot demonstrates more data points in the upper right portion of the plot than in the upper left. The most significant genes in the hippocampus had similar patterns of significance in both FC versus $\mathrm{N}$ (Fig. 2A) and CS versus $\mathrm{N}$ (Fig. 2B), whereas the $\mathrm{FC}$ versus $\mathrm{N}$ comparison in the amygdala (Fig. 2C) shows many more data points with $P$-values $<1 \mathrm{E}-6$ compared to the CS versus $\mathrm{N}$ comparison (Fig. 2D). The 50 most significantly regulated probe sets in FC mice compared to $\mathrm{N}$ mice according to $P$-value are presented for the hippocampus (Table 1) and amygdala (Table 2).

\section{$\mathrm{CS}$ and FC training produced similar gene regulation} in the hippocampus, but in the amygdala, FC training produced significantly greater levels of gene regulation compared to CS training

Expression levels in CS mice were highly similar to FC mice in the hippocampus (Fig. 3). This was not the case in the amygdala, where average FC versus $\mathrm{N}$ regulation in the amygdala was significantly greater than CS versus N (Fig. 3). Because most of the 50 most significant genes followed this expression pattern, the difference in FC regulation in the 50 most significantly regulated amygdala genes compared to the 50 most significantly regulated hippocampus genes was very highly significant $\left(P<10^{-5}\right.$, paired $t$-tests). We validated that our microarray results were accurate by testing expression levels for Fos, Dusp1, Nr4a1, Egr1, Junb, Gadd45b, Gadd45g, and Btg2 using quantitative real-time PCR (qPCR). For qPCR, tissue samples taken from independent training sessions were used ( $n=6$ for each group), and cDNA samples from both hippocampus and amygdala were prepared from single animals. For all genes tested, qPCR confirmed the upregulation following FC observed in our microarray data. Because most fold-change values were small in this experiment (see Tables 1 and 2; qPCR data are given in parentheses), we determined the geometric average of fold change and used paired $t$-tests on log scale data to determine the significance of group differences for the set of genes examined. According to qPCR, the hippocampal genes we examined were up-regulated $96 \%$ in CS and $85 \%$ in FC groups with no significant difference between these groups, whereas the genes we examined in the amygdala demonstrated $67 \%$ increase in expression in CS mice, while FC mice demonstrated a significantly greater $107 \%$ increase $(P<0.05)$.

\section{Transcription factor-binding site analysis identified promoter models that predict gene regulation with some regional specificity}

To account for the observed expression patterns, we generated promoter models based on the hypothesis that multiple transcription factors act coordinately to regulate many of the most significantly regulated genes. Transcription factor-binding sites were predicted for each of the 50 most significantly regulated genes in each brain region using previously described methods (Hannenhalli and Levy 2003). Twenty of the 50 most significantly regulated genes in the hippocampus were linked to all nine transcription factor-binding sites in the hippocampus model: CREB, E2F1, Pax4, Sp1, GATA1, AP2, ZF5, AP-1, and Nrf-1. Ten of the 50 most significantly regulated amygdala genes were linked to all 11 transcription factor-binding sites in the amygdala model: CREB, E2F1, Pax4, Sp1, GATA1, AP2, ZF5, Ets1, Elk1, Myc/ Max, and USF. To examine the accuracy of our transcription 
A. FC vs $\mathrm{N}$ Hippocampus

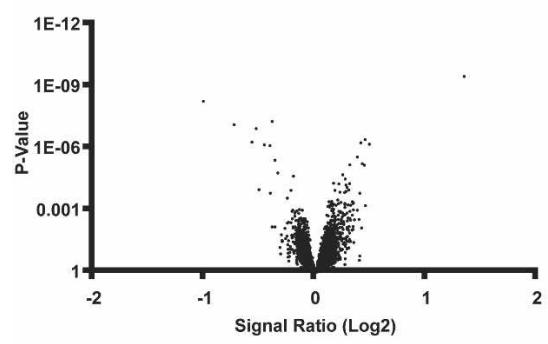

C. FC vs N Amygdala

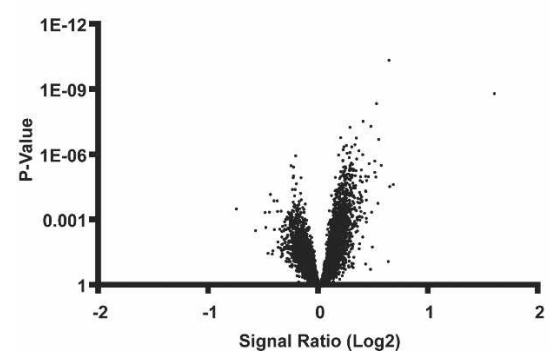

B. CS vs $\mathbf{N}$ Hippocampus

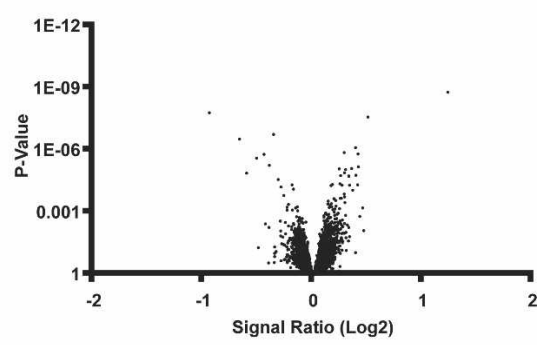

D. CS vs N Amygdala

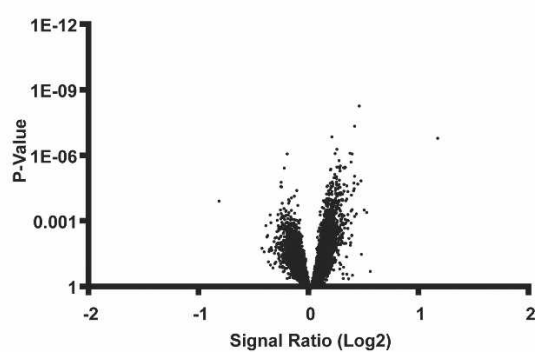

Figure 2. Volcano plots demonstrate the relationship between significance and Affymetrix signal ratios for gene regulation in the hippocampus and amygdala. Contrast $P$-values from a mixed model ANOVA are plotted in negative log scale on the $y$-axis against the base 2 log of the Affymetrix signal ratio for each probe set on the $x$-axis.

factor-binding site predictions, we examined six transcription factor-binding sites that were previously identified in the Fos promoter using Dnase1 protection assays (Shaw 1992; Lavrovsky et al. 1994; Schild-Poulter et al. 1996). We found that our annotation correctly identified the cAMP response element, the serum response element, and three out of four AP2-binding sites (data not shown). We then tested whether our transcription factorbinding site models could identify genes from the entire genome that were not among the genes used to produce the models, but were likely to be regulated in our data at lower levels of significance. High scoring matches for all nine binding sites identified in the hippocampal data were located upstream of 65 genes, and the expression of 32 of these genes had been measured by microarray. These 32 genes were significantly up-regulated in the hippocampus $(P=0.001)$ but regulation in the amygdala was not significant $(P=0.22)$ (Fig. 4$)$. The 11 transcription factors identified for the amygdala were located upstream of 59 genes, of which we had expression data for 29 genes. These 29 genes were significantly induced in the amygdala following classical fear conditioning $(P=0.003)$, but regulation was not significant in the hippocampus $(P=0.11)$ (Fig. 4$)$.

\section{Discussion}

Our study is the first to characterize gene regulation in the amygdala following classical conditioning using microarrays and to compare these changes to those observed in the hippocampus. Interestingly, we found that in the amygdala, changes in expression following $\mathrm{FC}$ versus $\mathrm{N}$ were greater than changes following CS versus N, whereas in the hippocampus, exposure to the CS or FC induced equivalent levels of gene regulation with respect to $\mathrm{N}$ mice. Our results are therefore consistent with proposed functions of the hippocampus and amygdala (Maren 2001), in which the hippocampus is involved in the configural component of the task, while the amygdala is involved in the associative component.

The similar transcriptional response to CS and FC training in the hippocampus indicates that nonassociative components of the task alone are sufficient to drive the most significant regulatory changes observed in fear-conditioned animals, with associative components of the task having no significant additional effect. This result is consistent with the proposed role of the hippocampus in the configural component of fear conditioning (Rudy and Wright-Hardesty 2005), but the changes in expression could also be correlated to other nonassociative components of the task, such as arousal. Similarly, nonassociative components of fear conditioning may occlude otherwise significant effects of associative learning on hippocampal gene regulation in our data. Additional experiments using immediate shock or pre-exposure to induce memory deficits would be required to determine the more precise behavioral correlates for each gene. For example, more precise experiments have been previously examined in the case of Fos (Radulovic et al. 1998). In those experiments, Fos expression increased in the hippocampus following CS exposure, but this regulation was dramatically inhibited by pre-exposure of the training stimuli, demonstrating that arousal associated with training was not correlated to Fos regulation. Similarly, cued associative learning (including shock) was not correlated to Fos induction in the hippocampus when contextual training was blocked by preexposure (Radulovic et al. 1998).

The graded levels of regulation in the amygdala may indicate that some cell populations of the amygdala are involved in the processing of CS and US, and that additional activity in these populations occurs during associative processes (Maren and Quirk 2004). Alternatively, the graded response may be due to gene regulation in additional nuclei of the amygdala when the CS is paired with the unconditioned stimulus. Although additional experiments would be required to determine which of these two possibilities is occurring for each gene in our data, the relevant experiment has been conducted for Fos, and in that experiment, Fos expression in the central nucleus was partially correlated with associative learning, but Fos expression in other nuclei correlated with other components of the task such as exposure to novelty (Radulovic et al. 1998).

We focused our studies on genes regulated by FC versus $\mathrm{N}$ because attempts to identify "associative-learning genes" in our data using FC versus CS comparisons were not successful. In the hippocampus, only the most significantly regulated gene, uridine monophosphate kinase, had a $P$-value $<10^{-3}$; however, that corresponded to a fold change of only $6 \%$. In the amygdala, JunB, FOSB, and nucleolin demonstrated significance of $P$-value $<10^{-3}$. The significance of JunB was accounted for in our examination of $\mathrm{FC}$ versus $\mathrm{N}$ genes. The differential regulation of FosB in FC versus N was validated, but the $11 \%$ FC versus CS fold change could not be validated by qPCR. Nucleolin demonstrated a change of only $9 \%$. Overall, the changes in expression demonstrated in FC versus CS in the amygdala included several of the regulatory changes identified in FC versus $\mathrm{N}$ studies and were more significant than FC versus CS changes in the hippocampus. For consistency with previous studies, we included a tone in our training protocol. Previous results indicate that this auditory cue may be processed by the hippocampus along with other elements 
Table 1. The 50 most significantly regulated probe sets in the hippocampus

\begin{tabular}{|c|c|c|c|c|c|c|}
\hline$P$-value (FC vs. $\mathrm{N}$ ) & Probe set & Symbol & Molecular role & $N\left(\log _{2}\right)$ & CS vs. $\mathrm{N}$ & FC vs. $\mathrm{N}$ \\
\hline \multicolumn{7}{|l|}{ Up-regulated genes } \\
\hline 3. $2 \mathrm{E}-10$ & 160901_at & Fos & DNA-binding transcription factor & 5.4 & $134(352) \%$ & $153(303) \%$ \\
\hline 3.7E-07 & 104598 at & Dusp1 & Phosphatase & 7.4 & $33(37) \%$ & $36(61 \%)$ \\
\hline $5.2 \mathrm{E}-07$ & 103501_at & Pura & DNA-binding transcription factor & 7.7 & $41 \%$ & $33 \%$ \\
\hline $6.1 \mathrm{E}-07$ & $102371_{\text {at }}$ & $\mathrm{Nr} 4 \mathrm{a} 1$ & DNA-binding transcription factor & 7.2 & $33(104) \%$ & $40(58) \%$ \\
\hline $2.6 \mathrm{E}-06$ & 98579_at & Egr1 & DNA-binding transcription factor & 8.4 & $27(55) \%$ & $30(40) \%$ \\
\hline $5.5 \mathrm{E}-06$ & 104510 at & Cacna2d1 & Voltage-dependent calcium channel & 6.7 & $31 \%$ & $34 \%$ \\
\hline $6.1 \mathrm{E}-06$ & $102363 \mathrm{r}$ at & Junb & DNA-binding transcription factor & 7.6 & $20(50) \%$ & $24(52) \%$ \\
\hline $6.3 \mathrm{E}-06$ & 103460_at & Ddit4 & Unknown & 7.1 & $28 \%$ & $36 \%$ \\
\hline $3.1 \mathrm{E}-05$ & 98782_at & Cplx2 & Binds syntaxin & 8.2 & $23 \%$ & $20 \%$ \\
\hline $4.8 \mathrm{E}-05$ & 94274 at & Ube2s & Ubiquitin carrier & 7.2 & $12 \%$ & $12 \%$ \\
\hline $5.0 \mathrm{E}-05$ & 102362_i_at & Junb & DNA-binding transcription factor & 7.6 & $22 \%$ & $24 \%$ \\
\hline $5.2 \mathrm{E}-05$ & 99597_at & Gnai2 & GTP binding & 8.4 & $22 \%$ & $16 \%$ \\
\hline $5.9 \mathrm{E}-05$ & 160617_at & Klf13 & DNA-binding transcription factor & 7.9 & $31 \%$ & $21 \%$ \\
\hline $7.8 \mathrm{E}-05$ & 102342 at & Nsf & Binds AMPA GluR2 & 10.3 & $25 \%$ & $22 \%$ \\
\hline $1.0 \mathrm{E}-04$ & $96497 \mathrm{~s}$ at & Myt1I & DNA-binding transcription factor & 5.5 & $22 \%$ & $23 \%$ \\
\hline $1.1 \mathrm{E}-04$ & 94405 at & Slc6a6 & Taurine transporter & 5.1 & $8 \%$ & $13 \%$ \\
\hline $1.2 \mathrm{E}-04$ & 93896_at & Ptprd & Protein tyrosine phosphatase & 6.3 & $25 \%$ & $21 \%$ \\
\hline $1.3 \mathrm{E}-04$ & 104663 at & Pip5k1b & Phosphatidylinositol 4-phosphate 5-kinase & 8.1 & $18 \%$ & $17 \%$ \\
\hline $1.4 \mathrm{E}-04$ & 93985_at & Tiparp & Amino acid ADP-ribosylation & 6.3 & $14 \%$ & $20 \%$ \\
\hline $1.5 \mathrm{E}-04$ & 103393 at & Pspc1 & RNA binding & 5.7 & $22 \%$ & $32 \%$ \\
\hline $3.1 \mathrm{E}-04$ & 92982_at & Bmp8a & Secreted signaling molecule & 5.2 & $15 \%$ & $15 \%$ \\
\hline 3.7E-04 & 160925 at & Nras & GTP binding & 6.5 & $13 \%$ & $11 \%$ \\
\hline $3.8 \mathrm{E}-04$ & 101930 at & Nfix & DNA-binding transcription factor & 9.2 & $33 \%$ & $26 \%$ \\
\hline 4.1E-04 & 99865 at & Bmpr2 & Receptor transmembrane serine/threonine kinase & 5.9 & $13 \%$ & $17 \%$ \\
\hline $4.2 \mathrm{E}-04$ & 160603_at & Pparbp & Transcriptional cofactor & 6.2 & $19 \%$ & $27 \%$ \\
\hline 4.3E-04 & 103243 at & Emp2 & Associates with $\beta(1)$ integrin & 7.5 & $9 \%$ & $11 \%$ \\
\hline 4.5E-04 & 99109_at & ler2 & Unknown & 5.1 & $20 \%$ & $27 \%$ \\
\hline 4.7E-04 & 92195 at & Cebpg & DNA-binding transcription factor & 4.8 & $15 \%$ & $20 \%$ \\
\hline 4.7E-04 & 104743_at & Cdh13 & Cell adhesion molecule & 5.8 & $22 \%$ & $15 \%$ \\
\hline $4.9 \mathrm{E}-04$ & $100446 r$ at & Sprr1b & Structural & 10.2 & $8 \%$ & $10 \%$ \\
\hline $5.0 \mathrm{E}-04$ & $102972 \mathrm{~s}$ at & Dab1 & Interacts with protein kinase pathways & 7.1 & $18 \%$ & $12 \%$ \\
\hline $5.1 \mathrm{E}-04$ & 98927_at & Rab6 & GTP binding & 10.1 & $26 \%$ & $20 \%$ \\
\hline $5.4 \mathrm{E}-04$ & 92703_at & Pb1 & Kinetochore-associated proteins & 6.3 & $19 \%$ & $23 \%$ \\
\hline $5.6 \mathrm{E}-04$ & 92899 at & Gad2 & $\gamma$-Aminobutyric acid (GABA) synthesis & 5.3 & $12 \%$ & $25 \%$ \\
\hline $5.9 \mathrm{E}-04$ & 100405 at & $\mathrm{Cb} \times 3$ & Binds heterochromatin & 6.6 & $37 \%$ & $37 \%$ \\
\hline $6.0 \mathrm{E}-04$ & 93740 at & Nsep1 & DNA-binding transcription factor & 10.8 & $10 \%$ & $9 \%$ \\
\hline $6.2 \mathrm{E}-04$ & 98914_at & Asf1a & Nucleosome component & 7.5 & $5 \%$ & $8 \%$ \\
\hline $6.8 \mathrm{E}-04$ & 99878 at & $\mathrm{Ddx} 10$ & RNA helicases & 4.1 & $8 \%$ & $9 \%$ \\
\hline $7.2 \mathrm{E}-04$ & 92362 at & Dusp8 & Phosphatase & 5.7 & $22 \%$ & $20 \%$ \\
\hline 8.7E-04 & 97682_r_at & Gstm3 & Transport/detoxification; major GST in brain & 7.9 & $10 \%$ & $11 \%$ \\
\hline $9.4 \mathrm{E}-04$ & 103288_at & Nrip1 & Transcriptional activity of the estrogen receptor & 6.8 & $14 \%$ & $27 \%$ \\
\hline \multicolumn{7}{|c|}{ Down-regulated genes } \\
\hline $5.0 \mathrm{E}-08$ & 161053 at & Ssty 1 & Unknown & 3.9 & $-22 \%$ & $-24 \%$ \\
\hline $1.1 \mathrm{E}-07$ & 103847 at & Ssty 2 & Unknown & 4.8 & $-27 \%$ & $-31 \%$ \\
\hline $4.9 \mathrm{E}-07$ & 94027 at & Cd84 & Cell adhesion & 7.6 & $-30 \%$ & $-33 \%$ \\
\hline $2.2 \mathrm{E}-05$ & 104581_at & Zdhhc6 & Metal binding & 3.7 & $-12 \%$ & $-13 \%$ \\
\hline $9.8 \mathrm{E}-05$ & 95705 s at & Actb & Cytoskeletal & 8.7 & $-34 \%$ & $-30 \%$ \\
\hline $1.1 \mathrm{E}-04$ & $97524 \mathrm{f}$ at & Amy 2 & Hydrolyze $1,4-\alpha$-qlucoside bonds & 4.1 & $-7 \%$ & $-14 \%$ \\
\hline $1.4 \mathrm{E}-04$ & 102818 at & $\mathrm{Xmr}$ & Unknown & 4.1 & $-19 \%$ & $-25 \%$ \\
\hline $2.5 \mathrm{E}-04$ & 94028_f_at & Cd84 & Cell adhesion & 5.1 & $-17 \%$ & $-16 \%$ \\
\hline $9.1 \mathrm{E}-04$ & M12481_M st & Actb & Cytoskeletal & 4.9 & $-14 \%$ & $-10 \%$ \\
\hline
\end{tabular}

Genes were ranked by contrast $P$-values from FC versus $\mathrm{N}$ group comparisons using mixed model ANOVA. Affymetrix probe set identifiers were matched to gene symbols, and molecular roles are reported. Affymetrix signal levels calculated by RMA are reported for naive $(\mathrm{N})$ mice along with percent change for FC versus $\mathrm{N}$ and $\mathrm{CS}$ versus $\mathrm{N}$ comparisons. qPCR values are given in parentheses.

of the context and may also be processed directly by the amydala through an alternate route that does not pass through the hippocampus (Maren 2001). The cue was therefore not expected to have any effect on hippocampal expression, other than to serve as a salient element of the novel environment. In the amygdala, the parallel processing of the cue could have induced parallel gene regulatory events in the amygdala. However, the cognitive correlates of these molecular events could still be classified as associative or nonassociative regardless of whether they are associated with cued or contextual conditioning.

Of the 50 most significantly regulated genes in our $\mathrm{FC}$ versus $\mathrm{N}$ data, 12 genes in the hippocampus and 11 genes in the amygdala had been previously described as being induced by some form of neural activity. Only two genes that we found to be up-regulated after fear conditioning (Fos and Egr1) were also identified in a previous study that examined gene regulation in two subregions of the hippocampus $1 \mathrm{~h}$ after classical fear conditioning (Levenson et al. 2004). The lack of overlap between these results may be due to the time points selected or the method used to obtain the tissue samples and prepare cDNA. Of the 50 most significant genes in each brain region from our data, six genes are common between these lists: Fos, Dusp1, Nr4a1, Egr1, Junb, and Ier2. Six genes shared between two top 50 lists would be highly unlikely based on chance $\left(\chi^{2}, P<0.001\right)$, suggesting that a cohort of genes is coordinately regulated in both brain regions. However, the divergence of these lists suggests that 
Table 2. The 50 most significantly regulated probe sets in the amygdala (FC vs. N)

\begin{tabular}{|c|c|c|c|c|c|c|}
\hline$P$-value (FC vs. $\mathrm{N}$ ) & Probe set & Symbol & Molecular role & $N\left(\log _{2}\right)$ & CS vs. $\mathrm{N}$ & FC vs. $\mathrm{N}$ \\
\hline \multicolumn{7}{|l|}{ Up-regulated genes } \\
\hline $3.8 \mathrm{E}-11$ & 102362_I_at & Junb & DNA-binding transcription factor & 7.11 & $32(7) \%$ & $55(63) \%$ \\
\hline $1.3 \mathrm{E}-09$ & 160901_at & Fos & DNA-binding transcription factor & 5.26 & $123(205) \%$ & $200(512) \%$ \\
\hline $3.8 \mathrm{E}-09$ & 102371_at & $\mathrm{Nr} 4 \mathrm{a} 1$ & DNA-binding transcription factor & 6.44 & $28(86) \%$ & $43(86) \%$ \\
\hline 4.2E-08 & 99109_at & ler2 & Unknown & 3.97 & $30 \%$ & $38 \%$ \\
\hline 4.7E-08 & 96318_at & ly6e & Unknown & 6.47 & $17 \%$ & $21 \%$ \\
\hline $1.4 \mathrm{E}-07$ & 98456_at & Stk19 & Serine/threonine kinase & 6.61 & $14 \%$ & $14 \%$ \\
\hline $1.5 \mathrm{E}-07$ & 101979_at & Gadd45g & Upstream activator of p38 and JNK MAPKs & 6.18 & $18(53) \%$ & $26(40) \%$ \\
\hline $1.7 \mathrm{E}-07$ & 98579_at & Egr1 & DNA-binding transcription factor & 7.98 & $32(66) \%$ & $45(44) \%$ \\
\hline $3.0 \mathrm{E}-07$ & 98878_r_at & Aaas & Nuclear pore/adapter & 5.42 & $21 \%$ & $25 \%$ \\
\hline $3.1 \mathrm{E}-07$ & 95726_at & Mlf2 & Unknown & 9.54 & $13 \%$ & $18 \%$ \\
\hline 3.7E-07 & 96554_r_at & Taf15 & DNA-binding transcription factor & 3.72 & $11 \%$ & $22 \%$ \\
\hline 3.7E-07 & 96872_at & Sgta & Unknown & 8.89 & $14 \%$ & $16 \%$ \\
\hline $5.0 \mathrm{E}-07$ & 104684_at & Grin1 & Ligand gated ion channel & 9.74 & $13 \%$ & $16 \%$ \\
\hline $5.7 \mathrm{E}-07$ & 103367_at & Galgt1 & Biosynthesis of glycosphingolipids & 6.98 & $24 \%$ & $28 \%$ \\
\hline $6.6 \mathrm{E}-07$ & 103277_s_at & Tnrc11 & Unknown & 6.23 & $18 \%$ & $21 \%$ \\
\hline $7.2 \mathrm{E}-07$ & 101487ffat & Ly6e & Unknown & 10.03 & $12 \%$ & $19 \%$ \\
\hline 8.6E-07 & 99872 s_at & Ftl1 & Iron binding & 8.70 & $29 \%$ & $31 \%$ \\
\hline $8.8 \mathrm{E}-07$ & 98429_at & Lypla2 & Lysophospholipase & 8.87 & $8 \%$ & $12 \%$ \\
\hline $1.1 \mathrm{E}-06$ & 92618_at & Serf2 & Unknown & 4.13 & $11 \%$ & $20 \%$ \\
\hline $1.6 \mathrm{E}-06$ & 92375_at & Ascc1 & DNA-binding transcription factor & 5.95 & $6 \%$ & $15 \%$ \\
\hline $1.6 \mathrm{E}-06$ & 104598_at & Dusp1 & Phosphatase & 6.87 & $29(55) \%$ & $41(95) \%$ \\
\hline 2.1E-06 & 102092_s_at & Ovgp1 & Unknown & 4.37 & $25 \%$ & $36 \%$ \\
\hline $2.6 \mathrm{E}-06$ & 161806_r_at & Atp6ap1 & Proton pump & 4.86 & $29 \%$ & $47 \%$ \\
\hline $2.7 \mathrm{E}-06$ & 104123_at & Fcho1 & Unknown & 6.77 & $18 \%$ & $21 \%$ \\
\hline $3.1 \mathrm{E}-06$ & 93126_at & $\mathrm{Ckb}$ & Creatine kinase & 11.20 & $16 \%$ & $17 \%$ \\
\hline $3.1 \mathrm{E}-06$ & 160503_at & $\mathrm{Fbl}$ & RNA binding & 7.56 & $12 \%$ & $16 \%$ \\
\hline $3.6 \mathrm{E}-06$ & 99014_at & Apbb1 & Unknown & 9.76 & $14 \%$ & $18 \%$ \\
\hline $3.8 \mathrm{E}-06$ & 96008_at & Dad1 & Unknown & 9.57 & $19 \%$ & $23 \%$ \\
\hline 4.3E-06 & 92987_at & Slc4a3 & Chloride/bicarbonate exchanger & 8.02 & $22 \%$ & $30 \%$ \\
\hline 4.4E-06 & 160242_at & Flcn & Unknown & 6.33 & $14 \%$ & $21 \%$ \\
\hline 4.4E-06 & 92714_at & Icam5 & Intercellular adhesion & 8.62 & $20 \%$ & $20 \%$ \\
\hline $4.5 \mathrm{E}-06$ & 100492_at & Ap2a2 & Tropomyosin-binding & 8.78 & $24 \%$ & $23 \%$ \\
\hline 4.7E-06 & 160215_at & Aes & Repressor of expression & 9.41 & $19 \%$ & $26 \%$ \\
\hline 5.0E-06 & 94918_at & Aars & tRNA synthase & 7.39 & $11 \%$ & $17 \%$ \\
\hline $5.2 \mathrm{E}-06$ & 101094_at & Hig1 & Unknown & 4.87 & $9 \%$ & $15 \%$ \\
\hline $5.2 \mathrm{E}-06$ & 93733_r_at & Rgs19ip1 & RGS protein degradation & 6.19 & $9 \%$ & $14 \%$ \\
\hline $6.6 \mathrm{E}-06$ & 101583_at & Btg2 & Regulates transcription & 8.07 & $12(87) \%$ & $20(204) \%$ \\
\hline $6.6 \mathrm{E}-06$ & 93618_at & Spnb3 & Binds Arp1 & 8.64 & $12 \%$ & $19 \%$ \\
\hline 7.1E-06 & 94780_at & Zbtb20 & DNA-binding transcription factor & 5.21 & $20 \%$ & $21 \%$ \\
\hline 7.1E-06 & 92622 at & Spin1 & Transporter & 7.82 & $15 \%$ & $16 \%$ \\
\hline 7.2E-06 & 97319_at & Rrad & G-protein & 5.75 & $9 \%$ & $18 \%$ \\
\hline 7.3E-06 & 93738_at & Slc2a1 & Glucose transporter & 6.59 & $13 \%$ & $16 \%$ \\
\hline 7.4E-06 & 101007_at & Mknk2 & Serine/threonine kinase & 6.21 & $13 \%$ & $23 \%$ \\
\hline 7.7E-06 & 102835_at & Ap2a2 & Lipid binding clathrin & 7.01 & $19 \%$ & $25 \%$ \\
\hline 8.1E-06 & 99486_at & Cenpb & Centromere binding & 6.74 & $23 \%$ & $21 \%$ \\
\hline $8.2 \mathrm{E}-06$ & 161666_f_at & Gadd45b & Upstream activator of p38 and JNK MAPKs & 4.92 & $31(21) \%$ & $33(52) \%$ \\
\hline \multicolumn{7}{|c|}{ Down-regulated genes } \\
\hline $9.5 \mathrm{E}-07$ & $92665 \mathrm{f}$ at & XIr & Unknown & 3.02 & $-6 \%$ & $-14 \%$ \\
\hline $2.8 \mathrm{E}-06$ & 161499_f_at & Rpl7I1 & Unknown & 4.77 & $-13 \%$ & $-17 \%$ \\
\hline 3.1E-06 & 103925_at & Mllt3 & DNA-binding transcription factor & 7.02 & $-15 \%$ & $-15 \%$ \\
\hline $8.2 \mathrm{E}-06$ & 94074_at & Fcgr3 & IgG binding & 4.97 & $-10 \%$ & $-15 \%$ \\
\hline
\end{tabular}

Genes were ranked by contrast $P$-values from FC versus $\mathrm{N}$ group comparisons using mixed model ANOVA. Affymetrix probe set identifiers were matched to gene symbols, and molecular roles are reported. Affymetrix signal levels calculated by RMA are reported for naive ( $\mathrm{N}$ ) mice along with percent change for $\mathrm{FC}$ versus $\mathrm{N}$ and $\mathrm{CS}$ versus $\mathrm{N}$ comparisons. qPCR values are given in parentheses.

additional cohorts of genes are being regulated in a brain-regionspecific manner. We used EASE analysis (Hosack et al. 2003), Ingenuity Pathway Analysis (http://www.ingenuity.com), and literature survey to identify gene functions for our lists of significantly regulated genes. Many of the genes identified in both the amygdala and hippocampus are categorized as DNA-binding transcription factors, immediate early proteins, or cell signaling genes (Tables 1, 2).

We identified groups of transcription factor-binding sites shared among the differentially expressed genes in the $5 \mathrm{kp}$ upstream of each gene (Hannenhalli and Levy 2003). The promoter models we thus generated successfully predicted regulation and also demonstrated tissue specificity. Because we were interested in building transcriptional models that include many transcription factor-binding sites that regulate cohorts of related genes (Harbison et al. 2004; Chesler et al. 2005), we used graph theoretical analysis to search for the largest group of transcription factor-binding sites that demonstrated complete linkage to the largest number of genes among each of our top 50 gene lists. In the hippocampus, our transcription factor-binding site model was used to predict 65 potentially regulated transcripts. In the amygdala, our model was used to predict 59 potentially regulated transcripts. Because our models rely on the assumption that coordinated binding of many transcription factors is occurring at each promoter, electromobility shift assays would not be appropriate to validate these results. Here we used a strategy in which 
A

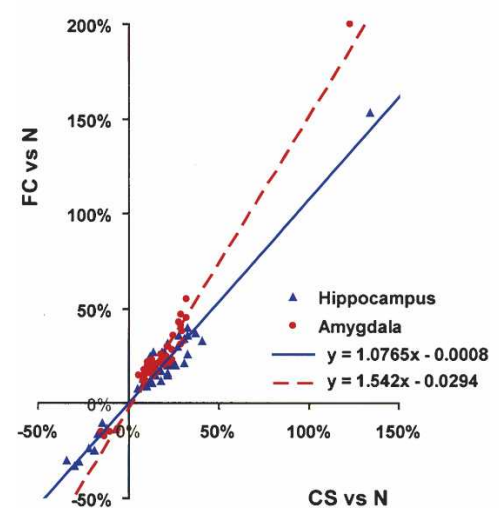

B

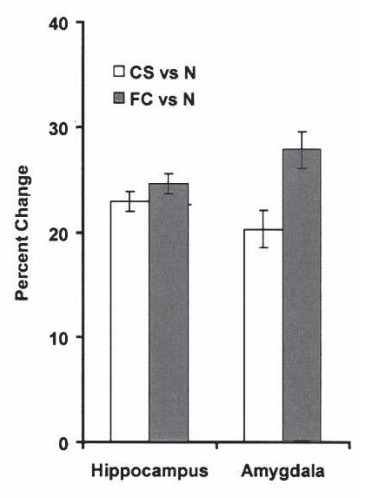

Figure 3. Gene regulation was equivalent following associative learning and CS exposure in the hippocampus, whereas gene regulation in the amygdala was greater following associative learning than nonassociative learning. (A) FC versus $\mathrm{N}$ regulation is plotted against $\mathrm{CS}$ versus $\mathrm{N}$ regulation for each of the top 50 genes in the hippocampus (blue triangles) and amygdala (red circles). Hippocampal genes were located near the identity function, as demonstrated by the slope of the regression line (blue solid line, slope $\left.=1.08, R^{2}=0.96\right)$. Amygdala genes were located along a line of greater slope (red dashed line, slope $\left.=1.54, R^{2}=0.96\right)$. $(B)$ Group statistics based on geometric averages across each set of 50 genes demonstrate that FC training in the hippocampus does not produce significantly greater regulation than CS training. In the amygdala, FC training does produce significantly greater regulation. Error bars represent SEM of pairwise changes.

changes in the expression of genes predicted by our models were examined in our existing microarray data. Although not all predicted genes were present on the array, the average expression values from 32 genes in the hippocampus and 29 genes in the amygdala demonstrated the expected pattern of up-regulation following classical fear conditioning, with some degree of regional specificity (Fig. 4). The overlap between regulatory modules generated for these two brain regions, including $C R E B, E 2 F 1$, Pax4, Sp1, GATA1, AP2, and ZF5, again suggests that common mechanisms are at work in both the hippocampus and amygdala, while our ability to predict gene regulation from these lists with some regional specificity supports the idea that additional brainregion-specific mechanisms exist. Brain-region-specific transcrip-
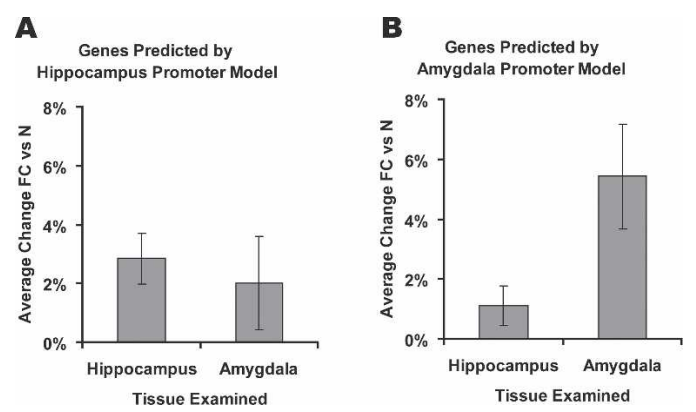

Figure 4. Transcription factor-binding site models predict regulation of additional genes for both amygdala and hippocampus with some specificity. (A) A set of transcription factor-binding sites identified from the 50 most significantly regulated hippocampal genes was detected in $32 \mathrm{ad}-$ ditional genes, which showed significant up-regulation in the hippocampus $(P<0.001)$, but up-regulation in the amygdala was not significant $(P>0.05)$. (B) A set of transcription factor-binding sites identified from the 50 most significantly regulated amygdala genes was detected in 27 additional genes, which showed significant up-regulation in the amygdala $(P<0.005)$, whereas up-regulation in the hippocampus was not significant $(P>0.05)$. tion factor-binding sites that together confer the specificity of the models include Ets1, Elk1, Myc/Max, and USF binding sites in the amygdala and Nrf1 and AP-1 in the hippocampus. It will be interesting to examine the role of these transcription factors in regionally specific gene regulation during memory storage. Chromatin immunoprecipitation (ChIP) from neural tissue following pharmacological manipulation has recently been accomplished (Kumar et al. 2005). By expanding these techniques to examine combinatorial binding of transcription factors using ChIP following behavioral manipulation, future experiments could address the hypotheses generated here. In future experiments, it will also be important to develop genetic and genomic approaches to identify the direct target genes of transcriptional regulators in vivo, as indirect effects are almost always possible with current molecular techniques.

To identify additional trans-acting genetic regulators, we used WebQTL (Wang et al. 2003; http://www.genenetwork.org) to search for gene cliques within our top 50 gene lists that were coordinately linked to genetic loci in an independent data set (Chesler et al. 2005). We found only one clique that was linked to two loci and contained genes that were regulated in both brain regions: Fos, Dusp1, Nr4a1, Ier2, Egr1, Junb, Gadd45b, and Btg2. One linkage locus on chromosome 12 contained 65 transcripts that included Myt1l, which was one of the 50 most significantly regulated genes in the hippocampus (Table 1 ) and was also regulated in the amygdala $(P<0.001)$. Another linkage locus on chromosome 18 contained $\sim 54$ transcripts that included Camk2a, a known regulator of immediate-early genes in response to neural activity (Colbran and Brown 2004). Future experiments would be required to determine how genetic variations in genes at these linkage sites, such as Myt1l and CamK2a, act to regulate the behaviorally responsive gene clique identified here.

Based on our results, we hypothesize that classical fear conditioning induces changes in the expression of a large battery of genes in both brain regions within $30 \mathrm{~min}$. Some of these genes may be regulated in both brain regions as a cohort that responds to a set of shared transcription factor-binding sites, while additional genes may be regulated by regionally specific involvement of particular transcription factor-binding sites, as has been observed during development (Davidson et al. 2002) and exposure to environmental stimuli (Harbison et al. 2004). Our main findings are that during classical fear conditioning, exposure to CS drives gene regulation in the hippocampus, while in the amygdala, genes are regulated partially by CS but are more greatly regulated by FC. Associative learning therefore correlated with gene regulation in the amygdala, while nonassociative components of classical fear conditioning correlated with gene regulation in both the amygdala and the hippocampus.

\section{Materials and Methods}

\section{Animals}

Six- to 8-wk-old male C57BL/6J mice were obtained from Jackson Labs (Bar Harbor, ME). Mice were housed under a 12-h light/12-h dark cycle (lights on at 7 a.m.) and allowed access to food and water ad libitum. All experiments were approved by the University of Pennsylvania Institutional Animal Care and Use Committee and performed in accordance with all National Institutes of Health guidelines. All mice were between 8 and 14 wk old at the time of behavioral training.

\section{Behavior}

Animals were individually housed and handled for $6 \mathrm{~d}$ prior to behavioral training. Handling consisted of removing mice from their home cage for $3 \mathrm{~min}$ while in the training room. Animals were always handled, trained, and tested at the same time of day (10:30 a.m. to 11:30 a.m.) to control for circadian effects. Experi-

\section{Learning \& Memory}


ments were performed in parallel. Naive $(\mathrm{N})$ mice were dissected between 11 a.m. and 12 p.m. on day 7. CS-exposed (CS) mice were exposed to the conditioning chamber for $3 \mathrm{~min}$. From 2:00 to $2: 30$ the mice were exposed to a tone $(2800 \mathrm{~Hz}, 85 \mathrm{~dB})$. CS mice were dissected $30 \mathrm{~min}$ after context/cue exposure. Fearconditioned (FC) mice were exposed to the same CS as CS mice, but additionally received a scrambled 2 -sec 1.5 -mA footshock from $2: 28$ to $2: 30$. FC mice were likewise dissected $30 \mathrm{~min}$ after training. Additional animals were given the same behavioral treatment in parallel, but were tested on day 8 . Testing consisted of re-exposure to the CS for $3 \mathrm{~min}$. Freezing behavior was scored by sampling at 5-sec intervals to determine the percentage of time that the mice spent in a stereotypical frozen posture. Groups of mice used for microarray analysis were trained in parallel with those tested behaviorally.

\section{Dissection}

Brains were rapidly dissected, placed in ice cold PBS for $10 \mathrm{sec}$, and then transferred to a mouse brain matrix (ASI Instruments). The matrix was occluded so that the brain rested at a consistent angle of $45^{\circ}$, and a $10-\mu \mathrm{m}$ blade was inserted at the posterior boundary of the circle of Willis. By following the $45^{\circ}$ angle of the brain matrix, we were able to expose the amygdalae without damaging the hippocampi. The amygdalae were dissected by cutting along the line of the external capsule following the angle of the lateral cortex from the surface of the amygdala to the bifurcation of the external capsule and then teasing the amygdala away from the optic tract, internal capsule, striatum, and other dorsal/medial structures. The resulting sample therefore included the cortical amygdaloid, basolateral, basomedial, lateral, central, and medial nuclei, but not the posterior portion of the amygdala. Hippocampi were dissected by teasing apart the posterior cerebral hemispheres and the lateral ventricles to unroll the posterior cerebrum. Hippocampi were carefully separated from the cortex, fimbria, and choroid plexus.

\section{RNA extraction, cDNA synthesis, and hybridization}

RNA extraction, cDNA synthesis, and hybridization were based on Affymetrix GeneChip Sample Cleanup Module (Mat. No. 1,020,407, 03/2002; Affymetrix) with some modifications. Briefly, RNA extraction was performed by homogenizing two hippocampi from one mouse or four amygdalae from two mice in $1 \mathrm{~mL}$ of Trizol reagent (Invitrogen) using a dounce homogenizer. Samples were extracted with $300 \mu \mathrm{L}$ of chloroform using spin-lock tubes (Eppendorf). Total RNA was precipitated with 1 $\mu \mathrm{L}$ of glycogen $(10 \mathrm{mg} / \mathrm{mL}), 1 / 10 \mathrm{vol}$ of $3 \mathrm{M} \mathrm{NaOAc}$, and $2 \mathrm{vol}$ of ethanol, then desalted with cold $80 \%$ ethanol, dried, and resuspended in sterile distilled water. RNA cleanup was performed using the RNeasy kit (Qiagen) according to instructions, followed by quantification by spectrophotometry. Synthesis of cDNA from purified total RNA was performed using SuperScript II (Invitrogen) according to instructions. Briefly, $5 \mu \mathrm{g}$ of total RNA was reverse-transcribed in $20-\mu \mathrm{L}$ reactions using $200 \mathrm{U}$ RT (SuperScript II). Temperature adjustment and first-strand synthesis were performed at $42^{\circ} \mathrm{C}$. Second-strand synthesis was performed using DNA ligase, Polymerase I, and RNase $\mathrm{H}$ from Escherichia coli (SuperScript II) according to instructions, followed by T4 DNA Polymerase to finish second-strand synthesis. Cleanup of doublestranded cDNA was performed using the GeneChip Sample Cleanup Module (Affymetrix) according to instructions. Synthesis and cleanup of biotin-labeled cRNA was performed by T7 polymerase in vitro transcription using the BioArray HighYield RNA Transcript Labeling Kit (Affymetrix). Ten microliters of cDNA was used for labeling reactions for each sample. Quantification of cRNA was performed by spectrophotometry, subtracting carryover cDNA from total concentration to obtain the concentration of labeled cRNA. Initial samples were analyzed by agarose gel electrophoresis stained with ethidium bromide. Fragmentation of $20 \mu \mathrm{g}$ of cRNA was performed by metalinduced hydrolysis using $5 \times$ fragmentation buffer supplied with the GeneChip Sample Cleanup Module (Affymetrix). The cRNA products were fragmented to $200 \mathrm{nt}$ or less, heated at $99^{\circ} \mathrm{C}$ for 5 min, and hybridized for $16 \mathrm{~h}$ at $45^{\circ} \mathrm{C}$ to Affymetrix mouse genome U74v2A microarrays by the Penn Microarray Facility. The microarrays were washed at low $(6 \times$ SSPE) and high $(100 \mathrm{mM}$ MES, $0.1 \mathrm{M} \mathrm{NaCl}$ ) stringency and stained with streptavidinphycoerythrin. Fluorescence was amplified by adding biotinylated anti-streptavidin and an additional aliquot of streptavidinphycoerythrin stain. A confocal scanner was used to collect fluorescence signal at $3 \mu \mathrm{m}$ resolution after excitation at $570 \mathrm{~nm}$. The average signal from two sequential scans was calculated for each microarray feature.

\section{Expression analysis}

Robust Multiarray Average (Bolstad et al. 2003) was used to compute intensity scores from image (.cel) files. Mixed model ANOVA was performed for each brain region using group (n, cs, fc) fixed variables and batch (1-4) random variables, and $P$-values were calculated from pairwise contrast calculations using Partek Pro software (Partek). Microarray data are available through GEO accession number GSE3963.

\section{Quantitative real-time PCR}

Quantitative PCR was performed according to the TIGR protocol for two-step RT PCR (Hegde et al. 2000) with some modifications. RNA extraction was performed by homogenizing two hippocampi from one mouse in $1 \mathrm{~mL}$ or two amygdalae from one mouse in $300 \mu \mathrm{L}$ of Trizol reagent (Invitrogen), using a dounce homogenizer. Samples were extracted with $1 / 3$ volume chloroform using spin-lock tubes (Eppendorf). Total RNA was precipitated with $1 \mu \mathrm{L}$ of glycogen $(10 \mathrm{mg} / \mathrm{mL}), 0.1 \mathrm{vol}$ of $3 \mathrm{M} \mathrm{NaOAc}$, and 2 vol of ethanol, then desalted with cold $80 \%$ ethanol, dried, and resuspended in sterile distilled water. RNA cleanup was performed using the RNeasy kit (Qiagen) according to instructions. DNase treatment and removal were performed with DNA-free (Ambion) according to instructions and resuspended in $100 \mu \mathrm{L}$ of sterile water. RNA precipitation was performed using $1 \mu \mathrm{L}$ of glycogen, $50 \mu \mathrm{L}$ of $7.5 \mathrm{M}$ ammonium hydroxide, and $250 \mu \mathrm{L}$ of ethanol, then desalted with cold $80 \%$ ethanol, dried, and resuspended in sterile distilled water and quantified by spectrophotometry at $260 \mathrm{~nm}$. To produce cDNA, $2 \mu \mathrm{g}$ of RNA from each sample was reverse-transcribed in $100-\mu \mathrm{L}$ reactions using Taqman RT reagents (ABI), according to instructions. Additional 2 - $\mu$ g RNA samples were prepared in parallel but without reverse transcriptase to act as a control for genomic contamination in quantitative PCR. Reverse-transcription conditions were $10 \mathrm{~min}$ at $25^{\circ} \mathrm{C}, 30 \mathrm{~min}$ at $48^{\circ} \mathrm{C}$, and $5 \mathrm{~min}$ at $95^{\circ} \mathrm{C}$ to heat-kill the enzyme. For each quantitative PCR reaction, $100-\mu \mathrm{L}$ RT reactions were diluted to $1500 \mu \mathrm{L}$ with sterile water, and $14 \mu \mathrm{L}$ of each sample was used for each qPCR reaction. qPCR was performed using $15 \mu \mathrm{L}$ of Quantitect SYBR master mix (Ambion) plus $0.5 \mu \mathrm{L}$ of each $10 \mu \mathrm{M}$ primer, pre-mixed and aliquoted for consistency. The efficiency of each primer set was determined for each of the genes selected (Fos, Junb, Nr4a1, Egr1, Dusp1, Gadd45b, Gadd45g, Btg2) using a serial dilution of pooled cDNA. All qPCR was performed in triplicate. Fos, Junb, Nr4a1, Egr1, Dusp1, and Gadd45b qPCR products were each cloned and sequenced, and all six cloned products contained the appropriate sequence. Primer sequences are available upon request. Threshold cycle $\left(C_{t}\right)$ values were adjusted for efficiency and then normalized to two internal control genes: HPRT and Actin $\gamma$. Median values from triplicate experiments were then used to generate group statistics. $P$-values were determined based on paired $t$-tests using log-scale values. Geometric averages were also calculated for each group.

\section{Transcription factor-binding site analysis}

TRANSFAC (Wingender et al. 1996) was used as a primary source of known binding profiles to annotate regions $5 \mathrm{~kb}$ upstream of all known RefSeq transcripts. This region was selected based on the fact that transcription factor-binding sites in several validated targets are localized in that region (Euskirchen et al. 2004). The annotation threshold for each position weight matrix was set so that the frequency of each PWM would be $1 / 50,000$ bp in 
nonconserved sequence and 1/5000 bp in conserved sequence, using human-mouse genome alignment from the UCSC database to determine conservation. The application of this method to the human genome has been previously described (Levy and Hannenhalli 2002). The upstream regions of the 50 most significantly regulated genes from each brain region were examined for each transcription factor-binding site, and the results were represented as a bipartite graph. All transcription factors from the largest completely connected subgraphs were combined to produce one promoter model for each brain region. For each model, genes that contain every site in the model were selected from genomic data.

\section{Acknowledgments}

We are grateful to John Tobias for helpful suggestions and training regarding data analysis and statistical methods and to Klaus Kaestner, Julie Blendy, Debu Chakravarti, and Lewis Chodosh for helpful suggestions regarding the organization and analysis of results. We are also thankful to Don Baldwin for microarray processing, data analysis training, and many helpful discussions during the course of this work. This work was supported by the National Institute on Aging R01AG018199, National Institute of Mental Health R01MH060244, and the David and Lucile Packard Foundation Fellowship in Science and Engineering.

\section{References}

Abel, T. and Lattal, K.M. 2001. Molecular mechanisms of memory acquisition, consolidation and retrieval. Curr. Opin. Neurobiol. 11: $180-187$.

Barrientos, R.M., O'Reilly, R.C., and Rudy, J.W. 2002. Memory for context is impaired by injecting anisomycin into dorsal hippocampus following context exploration. Behav. Brain Res. 134: 299-306.

Bernabeu, R., Bevilaqua, L., Ardenghi, P., Bromberg, E., Schmitz, P., Bianchin, M., Izquierdo, I., and Medina, J.H. 1997. Involvement of hippocampal cAMP/cAMP-dependent protein kinase signaling pathways in a late memory consolidation phase of aversively motivated learning in rats. Proc. Natl. Acad. Sci. 94: 7041-7046.

Bolstad, B.M., Irizarry, R.A., Astrand, M., and Speed, T.P. 2003. A comparison of normalization methods for high density oligonucleotide array data based on variance and bias. Bioinformatics 19: 185-193.

Bourtchouladze, R., Abel, T., Berman, N., Gordon, R., Lapidus, K., and Kandel, E.R. 1998. Different training procedures recruit either one or two critical periods for contextual memory consolidation, each of which requires protein synthesis and PKA. Learn. Mem. 5: 365-374.

Campeau, S., Hayward, M.D., Hope, B.T., Rosen, J.B., Nestler, E.J., and Davis, M. 1991. Induction of the c-fos proto-oncogene in rat amygdala during unconditioned and conditioned fear. Brain Res. 565: 349-352.

Cavallaro, S., Schreurs, B.G., Zhao, W., D'Agata, V., and Alkon, D.L. 2001. Gene expression profiles during long-term memory consolidation. Eur. J. Neurosci. 13: 1809-1815.

Cavallaro, S., D'Agata, V., Manickam, P., Dufour, F., and Alkon, D.L. 2002. Memory-specific temporal profiles of gene expression in the hippocampus. Proc. Natl. Acad. Sci. 99: 16279-16284.

Chesler, E.J., Lu, L., Shou, S., Qu, Y., Gu, J., Wang, J., Hsu, H.C., Mountz, J.D., Baldwin, N.E., Langston, M.A., et al. 2005. Complex trait analysis of gene expression uncovers polygenic and pleiotropic networks that modulate nervous system function. Nat. Genet. 37: 233-242.

Colbran, R.J. and Brown, A.M. 2004. Calcium/calmodulin-dependent protein kinase II and synaptic plasticity. Curr. Opin. Neurobiol. 14: $318-327$.

Davidson, E.H., Rast, J.P., Oliveri, P., Ransick, A., Calestani, C., Yuh, C.H., Minokawa, T., Amore, G., Hinman, V., Arenas-Mena, C., et al. 2002. A genomic regulatory network for development. Science $\mathbf{2 9 5}$ 1669-1678.

Donahue, C.P., Jensen, R.V., Ochiishi, T., Eisenstein, I., Zhao, M., Shors, T., and Kosik, K.S. 2002. Transcriptional profiling reveals regulated genes in the hippocampus during memory formation. Hippocampus 12: 821-833.

Euskirchen, G., Royce, T.E., Bertone, P., Martone, R., Rinn, J.L., Nelson, F.K., Sayward, F., Luscombe, N.M., Miller, P., Gerstein, M., et al. 2004. CREB binds to multiple loci on human chromosome 22. Mol. Cell. Biol. 24: 3804-3814.

Hannenhalli, S. and Levy, S. 2003. Transcriptional regulation of protein complexes and biological pathways. Mamm. Genome 14: 611-619.

Harbison, C.T., Gordon, D.B., Lee, T.I., Rinaldi, N.J., Macisaac, K.D., Danford, T.W., Hannett, N.M., Tagne, J.B., Reynolds, D.B., Yoo, J., et al. 2004. Transcriptional regulatory code of a eukaryotic genome. Nature 431: 99-104.

Hegde, P., Qi, R., Abernathy, K., Gay, C., Dharap, S., Gaspard, R., Hughes, J.E., Snesrud, E., Lee, N., and Quackenbush, J. 2000. A concise guide to cDNA microarray analysis. Biotechniques 29: 548-550, 552-554, 556 passim.

Hosack, D.A., Dennis Jr., G., Sherman, B.T., Lane, H.C., and Lempicki, R.A. 2003. Identifying biological themes within lists of genes with EASE. Genome Biol. 4: R70.

Irwin, L.N. 2001. Gene expression in the hippocampus of behaviorally stimulated rats: Analysis by DNA microarray. Brain Res. Mol. Brain Res. 96: 163-169.

Jeffery, K.J. and Hayman, R. 2004. Plasticity of the hippocampal place cell representation. Rev. Neurosci. 15: 309-331.

Kumar, A., Choi, K.H., Renthal, W., Tsankova, N.M., Theobald, D.E., Truong, H.T., Russo, S.J., Laplant, Q., Sasaki, T.S., Whistler, K.N., et al. 2005. Chromatin remodeling is a key mechanism underlying cocaine-induced plasticity in striatum. Neuron 48: 303-314.

Lavrovsky, Y., Abraham, N.G., Levere, R.D., Lavrovsky, V., Schwartzman, M.L., and Kappas, A. 1994. Characterization of a 142-bp fragment of the murine c-fos oncogene promoter upstream of the SIF-binding element. Gene 142: 285-290.

Leil, T.A., Ossadtchi, A., Cortes, J.S., Leahy, R.M., and Smith, D.J. 2002. Finding new candidate genes for learning and memory. J. Neurosci. Res. 68: 127-137.

Leil, T.A., Ossadtchi, A., Nichols, T.E., Leahy, R.M., and Smith, D.J. 2003. Genes regulated by learning in the hippocampus. J. Neurosci. Res. 71: 763-768.

Levenson, J.M., Choi, S., Lee, S.Y., Cao, Y.A., Ahn, H.J., Worley, K.C., Pizzi, M., Liou, H.C., and Sweatt, J.D. 2004. A bioinformatics analysis of memory consolidation reveals involvement of the transcription factor c-rel. J. Neurosci. 24: 3933-3943.

Levy, S. and Hannenhalli, S. 2002. Identification of transcription factor binding sites in the human genome sequence. Mamm. Genome 13: $510-514$.

Maren, S. 2001. Neurobiology of Pavlovian fear conditioning. Annu. Rev. Neurosci. 24: 897-931.

Maren, S. and Quirk, G.J. 2004. Neuronal signalling of fear memory. Nat. Rev. Neurosci. 5: 844-852.

Pare, D., Quirk, G.J., and Ledoux, J.E. 2004. New vistas on amygdala networks in conditioned fear. J. Neurophysiol. 92: 1-9.

Radulovic, J., Kammermeier, J., and Spiess, J. 1998. Relationship between fos production and classical fear conditioning: Effects of novelty, latent inhibition, and unconditioned stimulus preexposure. J. Neurosci. 18: 7452-7461.

Ressler, K.J., Paschall, G., Zhou, X.L., and Davis, M. 2002. Regulation of synaptic plasticity genes during consolidation of fear conditioning. $J$. Neurosci. 22: 7892-7902.

Robles, Y., Vivas-Mejia, P.E., Ortiz-Zuazaga, H.G., Felix, J., Ramos, X., and Pena de Ortiz, S. 2003. Hippocampal gene expression profiling in spatial discrimination learning. Neurobiol. Learn. Mem. 80: 80-95.

Rosen, J.B., Fanselow, M.S., Young, S.L., Sitcoske, M., and Maren, S. 1998. Immediate-early gene expression in the amygdala following footshock stress and contextual fear conditioning. Brain Res. 796: $132-142$.

Rudy, J.W. and Wright-Hardesty, K. 2005. The temporal dynamics of retention of a context memory: Something is missing. Learn. Mem. 12: $172-177$.

Schild-Poulter, C., Sassone-Corsi, P., Granger-Schnarr, M., and Schnarr M. 1996. Nucleosome assembly on the human c-fos promoter interferes with transcription factor binding. Nucleic Acids Res. 24: 4751-4758.

Sharp, P.E., McNaughton, B.L., and Barnes, C.A. 1985. Enhancement of hippocampal field potentials in rats exposed to a novel, complex environment. Brain Res. 339: 361-365.

Shaw, P.E. 1992. Ternary complex formation over the c-fos serum response element: p62TCF exhibits dual component specificity with contacts to DNA and an extended structure in the DNA-binding domain of p67SRF. EMBO J. 11: 3011-3019.

Stork, O., Stork, S., Pape, H.C., and Obata, K. 2001. Identification of genes expressed in the amygdala during the formation of fear memory. Learn. Mem. 8: 209-219.

Wang, J., Williams, R.W., and Manly, K.F. 2003. WebQTL: Web-based complex trait analysis. Neuroinformatics 1: 299-308.

Wingender, E., Dietze, P., Karas, H., and Knuppel, R. 1996. TRANSFAC: A database on transcription factors and their DNA binding sites. Nucleic Acids Res. 24: 238-241.

Received September 12, 2005; accepted in revised form December 6, 2005.

\section{Learning \& Memory}




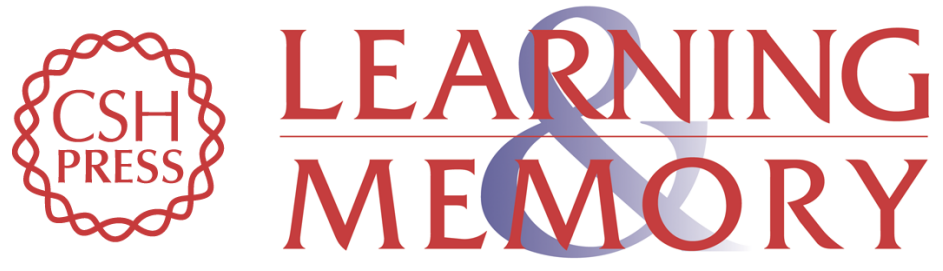

\section{Differential transcriptional response to nonassociative and associative components of classical fear conditioning in the amygdala and hippocampus}

Michael B. Keeley, Marcelo A. Wood, Carolina Isiegas, et al.

Learn. Mem. 2006, 13:

Access the most recent version at doi:10.1101//m.86906

References This article cites 39 articles, 10 of which can be accessed free at: http://learnmem.cshlp.org/content/13/2/135.full.html\#ref-list-1

License

Email Alerting

Receive free email alerts when new articles cite this article - sign up in the box at the Service top right corner of the article or click here. 